\title{
THE RING OF INTEGER-VALUED POLYNOMIALS OF A DEDEKIND DOMAIN
}

\author{
ROBERT GILMER, WILLIAM HEINZER, \\ DAVID LANTZ AND WILLIAM SMITH
}

(Communicated by Louis J. Ratliff, Jr.)

\begin{abstract}
Let $D$ be a Dedekind domain and $R=\operatorname{Int}(D)$ be the ring of integer-valued polynomials of $D$. We relate the ideal class groups of $D$ and $R$. In particular we prove that, if $D=\mathbf{Z}$ is the ring of rational integers, then the ideal class group of $R$ is a free abelian group on a countably infinite basis.
\end{abstract}

If $D$ is an integral domain with field of fractions $K$, the ring of integervalued polynomials of $D$ is denoted by $\operatorname{Int}(D)$ and is defined to be the subring of $K[t]$ (where $t$ is an indeterminate) consisting of those polynomials $f(t)$ in $K[t]$ such that $f(D) \subseteq D$. Work on rings of integer-valued polynomials has a long history. In particular, $\operatorname{Int}(\mathbb{Z})$, THE ring of integer-valued polynomials, has been studied at least since the work of Ostrowski [O] and Polya [P]. It was well known even then that $\operatorname{Int}(\mathbb{Z})$ is a free module over $\mathbb{Z}$, with a basis consisting of the binomial polynomials $B_{0}(t), B_{1}(t), \ldots$, where

$$
B_{n}(t)=\left(\begin{array}{l}
t \\
n
\end{array}\right)=t(t-1) \ldots(t-n+1) / n ! .
$$

Polya $[\mathrm{P}]$ gives a similar result with $\mathbb{Z}$ replaced by the ring of algebraic integers in a finite algebraic number field of class number 1 .

Polya showed that if the integral closure $D$ of $\mathbb{Z}$ in a finite algebraic number field is of class number 1 , then $\operatorname{Int}(D)$ is a free $D$-module with a basis consisting of one polynomial of each nonnegative degree. He called such a $D$-basis for $\operatorname{Int}(D)$ a "regular basis". His proof applies for any principal ideal domain $D$ of characteristic zero. Cahen $[\mathrm{Ca}, \S 2]$ proved that if $D$ is a Dedekind domain, $\operatorname{Int}(D)$ is a free $D$-module, and that if $D$ is a principal ideal domain, then $\operatorname{Int}(D)$ has a regular basis.

Received by the editors June 26,1989 . This research was presented to the 845 th meeting of the AMS, October 28-29, 1988, Lawrence, Kansas, by Professor Heinzer.

1980 Mathematics Subject Classification (1985 Revision). Primary 13B25, 13F05; Secondary 12B05.

Key words and phrases. ring of integer-valued polynomials, invertible ideals, Picard group.

The first and second authors gratefully acknowledge the support of the NSF.

The third author gratefully acknowledges the hospitality of Purdue University while this work was done. 
Brizolis [Br] has shown that, for a class of Dedekind domains $D$ including the ring of all algebraic integers in a finite algebraic number field, $\operatorname{Int}(D)$ is a Prüfer domain of Krull dimension two; McQuillan [Mc, § 5] and Chabert $[\mathrm{C} 2, \S 6]$ independently extended this result to all Dedekind domains $D$ with finite residue fields. Papers such as [GS], [GS2] and [C2] studied the nonzero finitely generated (i.e. invertible) ideals and the Picard group (i.e. the ideal class group, i.e. the factor group of the invertible fractional ideals by the subgroup of principal fractional ideals) of $\operatorname{Int}(D)$ for various domains $D$. The present paper gives a short exact sequence relating $\operatorname{Pic}(D)$ and $\operatorname{Pic}(\operatorname{Int}(D))$ for any Dedekind domain $D$. It allows us to conclude that $\operatorname{Pic}(\operatorname{Int}(\mathbb{Z}))$ is a free abelian group on a countably infinite basis.

Cahen and Chabert $[C C, \S 4]$ noted that $\operatorname{Int}(D)$ is often just $D[t]$, a condition closely related to finiteness of residue fields of $D$. Shibata, Sugatani and Yoshida [SSY] provided the explicit result that, for a Noetherian domain $D$, $\operatorname{Int}(D) \neq D[t]$ if and only if there is an associated prime ideal $P$ of a principal ideal of $D$ for which $D / P$ is a finite field. Thus the condition that the residue fields of $D$ be finite appears in many of the results below.

We freely use the fact that if $D$ is a Noetherian domain and $P$ is a prime ideal of $D$, then $\operatorname{Int}\left(D_{P}\right)=(\operatorname{Int}(D))_{D \backslash P}$. This was noted in [CC, p. 303], and a proof appears in [SSY, Proposition 4, p. 300]. Cahen and Chabert remark that this equality need not hold if $D$ is not Noetherian. Examples of such misbehavior appear in $[G]$, where examples are given of almost Dedekind domains $E$ with finite residue fields such that $\operatorname{Int}(E)$ is not a Prüfer domain. Any such domain $E$ contains a maximal ideal $M$ for which $\operatorname{Int}(E)_{E \backslash M}<\operatorname{Int}\left(E_{M}\right)$.

We frequently use the fact that any element of $\operatorname{Pic}(\operatorname{Int}(D))$ is represented by an integral ideal $I$ of $\operatorname{Int}(D)$ such that $I \cap D \neq(0)$. (McQuillan in [Mc, p. 162] calls such ideals I unitary; recall that an integral ideal of $\operatorname{Int}(D)$ is a fractional ideal contained in $\operatorname{Int}(D)$.) To see this, let $J$ be any invertible fractional ideal of $\operatorname{Int}(D)$, and denote by $K$ the field of fractions of $D$. Since $\operatorname{Int}(D)$ is contained in the principal ideal domain $K[t], J K[t]$ is principal, generated by a rational function $g(t)$. Hence $B=(g(t)) J$ generates the unit ideal of $K[t]$. Because $B$ is finitely generated, there exists a nonzero element $d$ of $D$ such that $I=d B \subseteq D[t] \subseteq \operatorname{Int}(D)$. The ideals $I$ and $J$ determine the same element of $\operatorname{Pic}(\operatorname{Int}(D))$, and because $I$ generates the unit ideal of $K[t]$, it follows that $I \cap D \neq(0)$. (We remark that this observation and statement (1) of Theorem 1 do not require that the domain be Dedekind; they are true for any integral domain $D$.)

Theorem 1. Let $D$ be a Dedekind domain, let $\alpha: \operatorname{Pic}(D) \rightarrow \operatorname{Pic}(\operatorname{Int}(D))$ be the natural map determined by extension of ideals, and let

$$
\beta: \operatorname{Pic}(\operatorname{Int}(D)) \rightarrow \prod_{P \in \max (D)} \operatorname{Pic}\left(\operatorname{Int}\left(D_{P}\right)\right)
$$

be the direct (Cartesian) product of the family of natural mappings Pic $(\operatorname{Int}(D)) \rightarrow$ $\operatorname{Pic}\left(\operatorname{Int}\left(D_{P}\right)\right)$, where $\max (D)$ denotes the set of maximal ideals of $D$. Then 
(1) $\alpha$ is injective,

(2) $\operatorname{Im}(\beta) \subseteq \bigoplus_{P \in \max (D)} \operatorname{Pic}\left(\operatorname{Int}\left(D_{P}\right)\right)$, and

(3) the sequence

$$
0 \rightarrow \operatorname{Pic}(D) \stackrel{\alpha}{\longrightarrow} \operatorname{Pic}(\operatorname{Int}(D)) \stackrel{\beta}{\longrightarrow} \bigoplus_{P \in \max (D)} \operatorname{Pic}\left(\operatorname{Int}\left(D_{P}\right)\right) \rightarrow 0
$$

of abelian groups is exact.

Proof. (1) Let $I$ be an invertible integral ideal of $D$ such that $I(\operatorname{Int}(D))=$ $f \cdot \operatorname{Int}(D)$ is principal. Applying the homomorphism $g \mapsto g(0)$ of $\operatorname{Int}(D)$ onto $D$ to the preceding equality, we see that $I=f(0) D$ is principal and, incidentally, that $f=f(0) \in I$. Therefore $\alpha$ is injective.

(2) Any element $x$ of $\operatorname{Pic}(\operatorname{Int}(D))$ is represented by an invertible integral ideal $J$ of $\operatorname{Int}(D)$ such that $J \cap D=I \neq(0)$. For all but a finite number of elements $P$ of $\max (D)$, we have $I \nsubseteq P$, and hence $I D_{P}=D_{P}$. For such $P$ we also have $J\left(\operatorname{Int}\left(D_{P}\right)\right)=\operatorname{Int}\left(D_{P}\right)$; consequently, $\beta(x)$ has only finitely many nonzero coordinates.

(3) We first show that the given sequence is exact in the middle. To see that $\operatorname{Im}(\alpha) \subseteq \operatorname{Ker}(\beta)$, choose an element $y$ of $\operatorname{Pic}(D)$ and an invertible integral ideal $J$ of $D$ that represents $y$. Then $J D_{P}$ is principal for each $P$ in $\max (D)$, and hence $J\left(\operatorname{Int}\left(D_{P}\right)\right)=J D_{P}\left(\operatorname{Int}\left(D_{P}\right)\right)$ is also principal. Therefore $\beta(\alpha(y))=0$, as we wished to show. For the converse, let $b \in \operatorname{Ker}(\beta)$ and let $B$ be an invertible integral ideal of $\operatorname{Int}(D)$ that represents $b$ and has the property that $C=B \cap D \neq(0)$. For each $P$ in $\max (D), B\left(\operatorname{Int}\left(D_{P}\right)\right)$ is principal, is generated by an element of $B$, and contains nonzero constant polynomials. Hence $B\left(\operatorname{Int}\left(D_{P}\right)\right)$ is generated by $C$, and the $D$-modules $B$ and $C(\operatorname{Int}(D))$ have the same localization at each maximal ideal of $D$. Therefore $B=C(\operatorname{Int}(D))$ and $b \in \operatorname{Im}(\alpha)$. We conclude that the given sequence is exact in the middle.

To complete the proof of (3), we show that $\beta$ is surjective. For this purpose it suffices to show that if $P$ is a maximal ideal of $D$ and if $J^{*}$ is an invertible integral ideal of $\operatorname{Int}\left(D_{P}\right)$ such that $J^{*} \cap D_{P} \neq(0)$, then there exists an invertible integral ideal $J$ of $\operatorname{Int}(D)$ for which $J\left(\operatorname{Int}\left(D_{P}\right)\right)=J^{*}$ and $J\left(\operatorname{Int}\left(D_{Q}\right)\right)=$ $\operatorname{Int}\left(D_{Q}\right)$ for each $Q$ in $\max (D) \backslash\{P\}$. We claim that $J=J^{*} \cap \operatorname{Int}(D)$ has the required properties. Since $J$ is the contraction to $\operatorname{Int}(D)$ of the ideal $J^{*}$ in the ring of fractions $\operatorname{Int}\left(D_{P}\right)$ of $\operatorname{Int}(D)$, we have $J\left(\operatorname{Int}\left(D_{P}\right)\right)=J^{*}$. Moreover, since $J^{*} \cap D_{P}$ is $P D_{P}$-primary, it follows that $J \cap D=J^{*} \cap D$ is $P$-primary. Thus for all $Q \neq P, J$ meets the multiplicative system $D \backslash Q$, so that $J\left(\operatorname{Int}\left(D_{Q}\right)\right)=\operatorname{Int}\left(D_{Q}\right)$.

It remains, finally, to show that $J$ is invertible. Since $J^{*}=J\left(\operatorname{Int}\left(D_{P}\right)\right)$ is finitely generated, there exists a finite subset $S$ of $J$ such that $S$ generates $J^{*}$. Also, $J \cap D=P^{k}$ for some positive integer $k$, and $P^{k}$ is finitely generated. If $J_{0}$ is the subideal of $J$ generated by $S \cup P^{k}$, then $J_{0}$ is finitely generated, $J_{0}\left(\operatorname{Int}\left(D_{Q}\right)\right)=J\left(\operatorname{Int}\left(D_{Q}\right)\right)=\operatorname{Int}\left(D_{Q}\right)$ for each $Q$ in $\max (D) \backslash\{P\}$, 
and $J_{0}\left(\operatorname{Int}\left(D_{P}\right)\right)=J\left(\operatorname{Int}\left(D_{P}\right)\right)=J^{*}$. From these equalities we conclude that $J=J_{0}$ is finitely generated and that $J$ is invertible since $J(\operatorname{Int}(D))_{D \backslash M}$ is principal for each $M$ in $\max (D)$.

Let $T$ be a totally ordered set with the interval topology, i.e. the smallest topology containing the sets $a^{+}=\{t \in T: t>a\}$ and $a^{-}=\{t \in T: t<a\}$ for all $a$ in $T$. Let $S$ be any topological space and $f, g: S \rightarrow T$ be continuous functions. Then the minimum function $\min (f, g): S \rightarrow T$ is again continuous. (For, $(\min (f, g))^{-1}\left(a^{+}\right)=f^{-1}\left(a^{+}\right) \cap g^{-1}\left(a^{+}\right)$and $(\min (f, g))^{-1}\left(a^{-}\right)=$ $f^{-1}\left(a^{-}\right) \cup g^{-1}\left(a^{-}\right)$.)

We denote by $\mathbb{Z}^{*}$ the totally ordered set $\mathbb{Z} \cup\{\infty\}$, with the interval topology; thus, singletons from the subset $\mathbb{Z}$ are open sets, and a base of neighborhoods of $\infty$ is given by the sets $[n, \infty]=\{m \in \mathbb{Z}: n \leq m\} \cup\{\infty\}$. Let $v$ be a discrete rank-one valuation on the field $K$. Then $K$ is a topological ring with neighborhood base at 0 given by the sets $U_{m}=\{a \in K: v(a) \geq m\}$ for $m$ in $\mathbb{Z}$. Let $f(t)=\sum_{i=0}^{n} b_{i} t^{i} \in K[t]$; we claim that the composite function $v \circ f: K \rightarrow \mathbb{Z}^{*}$ is continuous: For $a$ in $K$, suppose first that $f(a) \neq 0$. Choose the positive integer $m$ so that $m+v\left(b_{i} a^{j}\right)>v(f(a))$ for $i=1, \ldots, n$ and $j=0, \ldots, i-1$. For $c$ an element of $U_{m}, f(a+c)-f(a)$ is a sum of terms $\left(\begin{array}{l}i \\ j\end{array}\right) b_{i} c^{i-j} a^{j}$, where $1 \leq i \leq n$ and $0 \leq j \leq i-1$. Each of these terms has $v$-value greater than $v(f(a))$ by choice of $m$, and hence $v(f(a+c)-f(a))>$ $v(f(a))$. Therefore $v(f(a+c))=v(f(a))$. Now suppose $f(a)=0$, and consider a neighborhood $[n, \infty]$ of $\infty$. Choose the positive integer $m$ so that $m+v\left(b_{i} a^{j}\right)>n$ for $i=1, \ldots, n$ and $j=0, \ldots, i-1$. As above, $v(f(a+c))>$ $n$ for $c$ an element of $U_{m}$. Thus, in either case, for each neighborhood $V$ of $v(f(a)),(v \circ f)^{-1}(V)$ contains a neighborhood $a+U_{m}$ of $a$. By the last paragraph, if $f_{1}, \ldots, f_{k} \in K[t]$, then $\min \left(v \circ f_{1}, \ldots, v \circ f_{k}\right): K \rightarrow \mathbb{Z}^{*}$ is continuous.

For a topological space $S$, we denote by $F\left(S, \mathbb{Z}^{*}\right)$ the set of all continuous functions from $S$ into $\mathbb{Z}^{*}$. Note that, since $\mathbb{Z}^{*}$ is a topological semigroup under addition, $F\left(S, \mathbb{Z}^{*}\right)$ is a semigroup under pointwise addition. If a function $\phi$ in $F\left(S, \mathbb{Z}^{*}\right)$ does not take the value $\infty$, then $\phi$ is in the subgroup $F(S, \mathbb{Z})$. In this case, since $\mathbb{Z}$ is discrete, the sets $\phi^{-1}(n)$, for $n$ in $\mathbb{Z}$, form a disjoint open cover of $S$. Thus if $S$ is compact (not necessarily Hausdorff), then such a $\phi$ can assume only finitely many values. For any space $S$, the set $C(S, \mathbb{Z})$ of constant functions from $S$ into $\mathbb{Z}$ (i.e. those that assume only one value) is a subgroup of $F(S, \mathbb{Z})$.

Consider a discrete rank-one valuation domain $V$. As with the field $K$ above, $V$ is a topological ring with the topology induced by the valuation $v$. If $V$ is complete and has finite residue field, then $V$ is compact. (For, given a sequence $T_{0}$ of elements of $V$, since the residue field is finite, there exist an element $b_{0}$ of $T_{0}$ and a subsequence $T_{1}$ of $T_{0}$ for which $v\left(b-b_{0}\right)>0$ for all $b$ in $T_{1}$. Then there exist an element $b_{1}$ of $T_{1}$ and a subsequence $T_{2}$ of 
$T_{1}$ for which $v\left(b-b_{1}\right)>1$ for all $b$ in $T_{2}$. Continuing this process yields a Cauchy sequence $\left\{b_{n}\right\}$, which has a limit in the complete space $V$.)

2. Remark. The result in the preceding paragraph is a special case of a more general statement: Let $A$ be an ideal in the commutative ring $R$ with unity. Then the following conditions are necessary and sufficient for $R$ to be compact Hausdorff in the $A$-adic topology: (1) $A$ is finitely generated. (2) The factor ring $R / A$ is finite. (3) The powers of $A$ intersect in the zero ideal. An extension of the argument in the last paragraph proves the sufficiency of these conditions, and the necessity of (3) is clear and that of (2) is not difficult to see. For the necessity of (1), note first that if $R$ is compact Hausdorff, then $R$ is complete and $R / A^{2}$ is also finite, so that Theorem 8.4 of [Ma] shows that $A$ is finitely generated.

Now suppose the discrete rank-one valuation domain $V$ has finite residue field but is not necessarily equal to its completion $\hat{V}$. Let $p$ be a generator of the maximal ideal of $V$. By [C2, § 2], there is a bijection from $\hat{V}$ onto $S=\operatorname{Spec}(\operatorname{Int}(V) / p \operatorname{Int}(V))$, given by associating to the element $a$ of $\hat{V}$ the prime ideal $M_{a} / p \operatorname{Int}(V)$, where $M_{a}=\{f \in \operatorname{Int}(V): f(a) \in p \hat{V}\}$. In fact, if $S$ is considered as a topological space under its Zariski topology, this bijection is a homeomorphism: To see that it is continuous, take a basic closed subset of the spectrum, i.e. the set of ideals $M_{a} / p \operatorname{Int}(V)$ (for elements $a$ of $\hat{V}$ ) that contain a given ideal $I / p \operatorname{Int}(V)$, or equivalently, the set of ideals $M_{a} / p \operatorname{Int}(V)$ for which $v(f(a)) \geq 1$ for every $f$ in $I$; its inverse image in $\hat{V}$ is $\bigcap_{f \in I} \hat{V} \backslash(v \circ f)^{-1}\left(1^{-}\right)$, a closed set. Now since $\hat{V}$ is compact and $S$ is Hausdorff (the latter because $\operatorname{Int}(V) / p \operatorname{Int}(V)$ is zero-dimensional), it follows that the bijection is a homeomorphism.

We have just mentioned that the ring $\operatorname{Int}(V) / p \operatorname{Int}(V)$ is zero-dimensional. In fact, it is von Neumann regular. To see this, we only need to note that it is reduced, and this holds in a quite general setting: Let $r$ be a nonzero element of the domain $D$ for which $r D$ is a radical ideal of $D$; then we claim $\operatorname{rInt}(D)$ is a radical ideal of $\operatorname{Int}(D)$. For, if $f \in \operatorname{Int}(D)$ and $f^{k} \in \operatorname{rInt}(D)$ for some $k$, then $(f(a))^{k} \in r D$ for every $a$ in $D$, so $f(a) \in r D$ for every $a$ in $D$; thus, $f / r \in \operatorname{Int}(D)$ and $f \in r \operatorname{Int}(D)$, as we wished to show.

Theorem 3. Let $V$ be a discrete rank-one valuation domain with finite residue field. Denote its completion by $\hat{V}$. Then Pic $(\operatorname{Int}(V))$ is isomorphic to the factor group $F(\hat{V}, \mathbb{Z}) / C(\hat{V}, \mathbb{Z})$.

Proof. Assume that the element $p$ of $V$ generates the maximal ideal of $V$. Let $q=|V / p V|$, and denote by $v$ the normed valuation associated with $V$. Let $\{u(i)\}_{i=0}^{q-1}$ be a complete set of residues of $p V$ in $V$, and for the positive integer $n$ with $q$-adic expansion $n=a_{0}+a_{1} q+\ldots+a_{r} q^{r}$, where $0 \leq a_{i} \leq q-1$, let $s_{n}=u\left(a_{0}\right)+u\left(a_{1}\right) p+\ldots+u\left(a_{r}\right) p^{r}$. We let $f_{0}(t)=1$ and $f_{n}(t)=\prod_{i=0}^{n-1}\left(t-s_{i}\right)$ for $n>0$, and we let $g_{n}(t)=f_{n}(t) / p^{k(n)}$, where $k(n)=\sum_{i=0}^{n}\left[n / q^{i}\right]$ and [·] 
denotes the greatest integer function. It is known [C1, Lemma 1] (see also [G, Proposition 9]) that $\left\{g_{n}(t)\right\}_{n=0}^{\infty}$ is a free $V$-module basis for $\operatorname{Int}(V)$. Moreover, since $p$ generates the maximal ideal of $\hat{V}$ and since $\{u(i)\}_{i=0}^{q-1}$ is also a complete set of residues of $p \hat{V}$ in $\hat{V}$, the same result shows that $\left\{g_{n}(t)\right\}_{n=0}^{\infty}$ is a free $\hat{V}$-module basis for $\operatorname{Int}(\hat{V})$. In particular, $\operatorname{Int}(V) \subseteq \operatorname{Int}(\hat{V})$ in this case. Let $I$ be a finitely generated integral ideal of $\operatorname{Int}(V)$ that meets $V$ nontrivially. For each $d$ in $\hat{V}, I(d)=\{f(d): f \in I(\operatorname{Int}(\hat{V}))\}$ is a nonzero ideal of $\hat{V}$. Since $v(I(d))$ is the minimum value of $v(f(d))$ as $f$ varies over a finite set of generators, we see that $v(I(\cdot)): \hat{V} \rightarrow \mathbb{Z}^{*}$ is a continuous function with image contained in $\mathbb{Z}$; similar reasoning shows that $v(I J(\cdot))=v(I(\cdot))+v(J(\cdot))$, for if $\left\{f_{i}\right\}_{1}^{n}$ generates $i$, if $\left\{g_{j}\right\}_{1}^{m}$ generates $J$, if $v(I(d))=v\left(f_{i^{*}}(d)\right)$ and $v(J(d))=v\left(g_{j^{*}}(d)\right)$, then it is easy to show that $v(I J(d))=v\left(f_{i^{*}}(d) \cdot g_{j^{*}}(d)\right)=v\left(f_{i^{*}}(d)\right)+v\left(g_{j^{*}}(d)\right)=v(I(d))+v(J(d))$. In particular, $v(I(\cdot)) \in F(\hat{V}, \mathbb{Z})$.

By [Mc, Theorem 5.3, page 175], if $I, J$ are finitely generated (and hence invertible) integral ideals of $\operatorname{Int}(V)$ that meet $V$ nontrivially, and if, for each $d$ in $\hat{V}$, the $\hat{V}$-ideals $I(d)$ and $J(d)$ are equal, then $I=J$. Thus the mapping $I \mapsto v(I(\cdot))$ from the set of invertible integral ideals of $\operatorname{Int}(V)$ meeting $V$ nontrivially into $F(\hat{V}, \mathbb{Z})$ is injective. Any principal integral ideal of $\operatorname{Int}(V)$ meeting $V$ nontrivially is generated by a power $p$, so multiplying $I$ by a principal ideal changes the function $v(I(\cdot))$ by adding a constant function. Moreover, every invertible fractional ideal of $\operatorname{Int}(V)$ is a principal multiple of an integral ideal meeting $V$ nontrivially. Hence there is an injective mapping $h$ of $\operatorname{Pic}(\operatorname{Int}(V))$ into $F(\hat{V}, \mathbb{Z}) / C(\hat{V}, \mathbb{Z})$, determined by $I \mapsto v(I(\cdot))$, and because $v(I J(\cdot))=v(I(\cdot))+v(J(\cdot))$, the mapping $h$ is a homomorphism.

To see that this mapping is surjective, it is enough to show that any continuous bounded function $\phi$ from $\hat{V}$ into the set of nonnegative integers is $v(I(\cdot))$ for some invertible integral ideal $I$ of $\operatorname{Int}(V)$ meeting $V$ nontrivially. Such a $\phi$ has the form $\phi=\chi_{1}+2 \chi_{2}+\ldots+m \chi_{m}$, where $\chi_{i}$ is the characteristic function of the set $\phi^{-1}(i)$, and these sets form a partition of $\hat{V}$ into clopen (closed and open) sets. So it is enough to show that the characteristic function of a clopen set in $\hat{V}$ is $v(I(\cdot))$ for some $I$. We use the natural embedding of the spectrum $S$ of the von Neumann regular ring $\operatorname{Int}(V) / p \operatorname{Int}(V)$ as a subspace of $\operatorname{Spec}(\operatorname{Int}(V))$ and the homeomorphism from the paragraph following Remark 2. A clopen set $U$ in $S$ is the set of ideals $M_{a}$ containing an ideal $I$ generated by $p$ and an element that is idempotent modulo $\operatorname{pInt}(V)$; thus $M_{a} \in U$ iff $I \subseteq M_{a}$ iff $I(a) \subseteq p \hat{V}$ iff $v(I(a)) \geq 1$ iff $v(I(a))=1$ (since $p \in I$ ). It follows that $v(I(\cdot))$ is the characteristic function of $U$. This completes the proof.

Remark 4. [CC, Example 5, p. 303][Mc, Proposition 2.8, p. 166][SSY, Theorem 2, p. 298]. Let $V$ be a discrete rank-one valuation domain with infinite residue field. Then $\operatorname{Int}(V)=V[t]$, a unique factorization domain, so $\operatorname{Pic}(\operatorname{Int}(V))$ is trivial. 
Lemma 5 [Ca, Lemma, p. 751]. Let $D$ be a Dedekind domain, and let $q$ be a positive integer. Then the set of maximal ideals $P$ of $D$ for which $D / P$ is a field of $q$ elements is finite. Therefore the set of maximal ideals $P$ of $D$ for which $D / P$ is a finite field is countable.

Proof. The statement is clear if $D$ is a field. If $D$ is not a field, let $x$ be a nonzero nonunit in $D$. Then for any maximal ideal $P$ for which $D / P$ is a field of $q$ elements, $x\left(x^{q-1}-1\right)=x^{q}-x \in P$, so $x \in P$ or $x^{q-1}-1 \in P$. Since neither $x$ nor $x^{q-1}-1$ is zero, each is in only finitely many maximal ideals $P$.

Theorem 6. Let $D$ be a countable Dedekind domain. If at least one of the residue fields at maximal ideals of $D$ is finite, then Pic $(\operatorname{Int}(D))$ is the direct sum of $P i c(D)$ and a countably infinite number of copies of $\mathbb{Z}$.

Proof. Let $P$ be a maximal ideal for which $D / P$ is finite. By the proof of Theorem 3, a continuous bounded function from the completion $V$ of $D_{P}$ into $\mathbb{Z}$ is the minimum of a finite set of compositions of the valuation associated with $V$ and polynomials with coefficients in the field of fractions of $D$. Since there are only countably many such polynomials, $F(V, \mathbb{Z})$ is a countable subgroup of the direct product of copies of $\mathbb{Z}$ indexed by $V$. By [F, Theorem 19.2, p. 94], $F(V, \mathbb{Z})$ is a free abelian group. The constant function 1 is the generator of $C(V, \mathbb{Z})$, and it can be chosen as one of a set of free generators of $F(V, \mathbb{Z})$ (because if $1=\sum_{i} a_{i} e_{i}$ for some basis elements $e_{i}$ and integers $a_{i}$, then the $a_{i}$ 's are relatively prime, so there is a basis of $F(V, \mathbb{Z})$, or even just of the subgroup generated by the finitely many $e_{i}$ 's in the sum, containing 1 ; see [Ba, p. 166], [F, Lemma 9.1]). Thus $F(V, \mathbb{Z}) / C(V, \mathbb{Z})$ is again a free abelian group. Its basis is clearly countable. But it cannot be finite; for, suppose $F(V, \mathbb{Z})$ were generated by the finite set $\left\{f_{i}\right\}_{i=1}^{n}$. Each $f_{i}$ induces a finite partition $V=B_{i 1} \cup \ldots \cup B_{i k(i)}$ of $V$ into clopen sets such that the restriction of $f_{i}$ to each $B_{i j}$ is a constant function. Form all possible sets of the form $C=\bigcap_{i=1}^{n} B_{i j}$ for $1 \leq j \leq k(i)$. There are only finitely many such sets $C$, each is clopen, each $f_{i}$ is constant-valued on each $C$, and the sets $C$ form a partition of $V$. Because $\left\{f_{i}\right\}_{1}^{n}$ generates $F(V, \mathbb{Z})$, each $f$ in $F(V, \mathbb{Z})$ is constant-valued on each of the sets $C$. Hence if there are $k$ such nonempty sets $C$, then each $f$ in $F(V, \mathbb{Z})$ assumes at most $k$ distinct values. This is a contradiction, for the cosets of $p^{r} V$ form a partition of $V$ into clopen subsets, and an element of $F(V, \mathbb{Z})$ can assume an arbitrary value on each of these cosets.

Now by Remark 4 and Lemma 5, $\bigoplus_{P \in \max (D)} \operatorname{Pic}\left(\operatorname{Int}\left(D_{P}\right)\right)$ is still a free abelian group on a countably infinite basis, so the short exact sequence of Theorem 1 splits, and the result follows.

Corollary 7. Pic $(\operatorname{Int}(\mathbb{Z}))$ is a free abelian group on a countably infinite basis. More generally, if $D$ is any countable PID with at least one finite residue field, then Pic $(\operatorname{Int}(D))$ is a free abelian group on a countably infinite basis. 
Remark 8. Corollary 7 extends to a similar conclusion for the Grothendieck group $K_{0}(\operatorname{Int}(D))$. In fact, the following more general result is true: If $E$ is a Dedekind domain with the property that $\operatorname{Int}(E)$ is Prüfer (or equivalently, by [C2], that each residue field is finite), then $K_{0}(\operatorname{Int}(E)) \simeq \mathbb{Z} \oplus \operatorname{Pic}(\operatorname{Int}(E))$. So by Theorem 6, $K_{0}(\operatorname{Int}(E))$ is the direct sum of $\operatorname{Pic}(E)$ and a free abelian group of countably infinite rank. To see that $K_{0}(\operatorname{Int}(E)) \simeq \mathbb{Z} \oplus \operatorname{Pic}(\operatorname{Int}(E))$, we show first that $\operatorname{Int}(E)$ has the Steinitz property, i.e. $I \oplus J \simeq \operatorname{Int}(E) \oplus I J$ for any invertible ideals $I, J$ of $\operatorname{Int}(E)$. To do so, as noted above, we may assume that $I$ and $J$ are integral ideals meeting $E$ nontrivially. Then there is a nonzero element $a$ of $I \cap J \cap E$. By [C2], there exist elements $f$ and $g$ of $\operatorname{Int}(E)$ for which $I=(a, f)$ and $J=(a, g)$. Define a homomorphism $\varphi: \operatorname{Int}(E) \rightarrow I / I J$ by $\varphi(h)=h a+I J$ and set $L=\operatorname{ker}(\varphi)$. Since $\varphi$ is a surjection, there are two short exact sequences

$$
0 \rightarrow L \rightarrow \operatorname{Int}(E) \rightarrow I / I J \rightarrow 0
$$

and

$$
0 \rightarrow I J \rightarrow I \rightarrow I / I J \rightarrow 0 .
$$

Since, by hypothesis, all finitely generated ideals are invertible (projective), we can apply Schanuel's lemma to conclude that $I \oplus L \simeq I J \oplus \operatorname{Int}(E)$. Taking the second exterior power, we have $I L \simeq I J$ and hence $L \simeq J$. This produces the Steinitz condition $I \oplus J \simeq I J \oplus \operatorname{Int}(E)$. Since $\operatorname{Int}(E)$ is Prüfer, a finitely generated torsion-free $\operatorname{Int}(E)$-module $P$ is isomorphic to a direct sum $I_{1} \oplus$ $\ldots \oplus I_{k}$ of invertible ideals (cf. [K, Theorem 1]), and hence, using the Steinitz property, $P$ is isomorphic to $F \oplus I_{1} \cdots I_{k}$, where $F$ is a free $\operatorname{Int}(E)$-module of rank $k-1$. It follows that $K_{0}(\operatorname{Int}(E)) \simeq \mathbb{Z} \oplus \operatorname{Pic}(\operatorname{Int}(E))$. (See also [Ba, Proposition 3.7, p. 468]. J. Brewer and L. Klingler have independently observed that $\operatorname{Int}(E)$ has the Steinitz property.)

\section{REFERENCES}

[Ba] H. Bass, Algebraic K-theory, W. A. Benjamin, New York, 1968.

[Br] D. Brizolis, $A$ theorem on ideals in Prüfer rings of integral-valued polynomials, Comm. in Algebra 7 (1979), 1065-1077.

[Ca] P.-J. Cahen, Polynomes a valeurs entiers, Canad. J. Math. 24 (1972), 747-754.

[CC] P.-J. Cahen and J.-L. Chabert, Coefficients et valeurs d'un polynôme, Bull. Sc. Math. (2nd series) 95 (1971), 295-304.

[C1] J.-L. Chabert, Anneaux de "polynômes à valeurs entières" et anneaux de Fatou, Bull. Soc. math. France 99 (1971), 273-283.

[C2] _ Un anneau de Prüfer, J. Algebra 107 (1987), 1-16.

[F] L. Fuchs, Infinite Abelian Groups, vol. I, Academic Press, New York/London, 1970.

[G] R. Gilmer, Prüfer domains and rings of integer-valued polynomials, J. Algebra (to appear).

[GS1] R. Gilmer and W. Smith, Finitely generated ideals of the ring of integer-valued polynomials, J. Algebra 81 (1983), 150-164.

[GS2] _ Integer-valued polynomials and the strong two-generator property, Houston J. Math. 11 (1985), 65-74. 
[GM] H. Gunji and D. L. McQuillan, On a class of ideals in an algebraic number field, J. Number Theory 2 (1970), 207-222.

[K] I. Kaplansky, Modules over Dedekind rings and valuation rings, Trans. Amer. Math. Soc. 72 (1952), 327-340.

[Ma] H. Matsumura, Commutative ring theory, Cambridge University Press, Cambridge, 1986.

[Mc] D. McQuillan, On Prüfer domains of polynomials, J. Reine Angew. Math. 358 (1985), 162-178.

[O] A. Ostrowski, Über ganzwertige Polynome in algebraischen Zahlkörpern, J. Reine Angew. Math. 149 (1919), 117-124.

[P] G. Polya, Über ganzwertige Polynome in algebraischen Zahlkörpern, J. Reine Angew. Math. 149 (1919), 97-116.

[SSY] F. Shibata, T. Sugatani, and K. Yoshida, Note on rings of integral-valued polynomials, C. R. Math. Rep. Acad. Sci. Canada 8 (1986), 297-301.

[Z] H. Zantema, Integer valued polynomials over a number field, manuscr. math. 40 (1982), 155-203.

Department of Mathematics, Florida State University, Tallahassee, Florida 32306

Department of Mathematics, Purdue University, West Lafayette, Indiana 47907

Department of Mathematics, Colgate University, Hamilton, New York 13346

Department of Mathematics, University of North Carolina at Chapel Hill, Chapel Hill, North Carolina 27599 